\title{
Oral spindle cell lipomas
}

\author{
Patrícia C. Caldeira ${ }^{1}$, Vanessa F. Bernardes ${ }^{1}$, Alessandra C. Miranda ${ }^{1}$, Déborah C. Telles ${ }^{2}$, \\ Rodrigo M. R. Batista ${ }^{3}$, Carlos A. Ribeiro ${ }^{4}$, Tarcília A. Silva ${ }^{1}$
}

\footnotetext{
${ }^{1}$ Department of Oral Pathology and Surgery, School of Dentistry, Universidade Federal de Minas Gerais, Belo Horizonte, Brazil;

${ }^{2}$ Hospital of Clinics, Universidade Federal de Minas Gerais, Belo Horizonte, Brazil;

${ }^{3}$ Association against Cancer of the Midwest Minas Gerais, Divinópolis, Brazil;

${ }^{4}$ Roberto Alvarenga Institute, Belo Horizonte, Brazil.

Email: tarcilia@ufmg.br
}

Received 9 September 2011; revised 3 November 2011; accepted 24 November 2011.

\begin{abstract}
Spindle cell lipoma is an histological type of lipoma which are rarely found in the oral cavity. We describe two cases of intraoral spindle cell lipomas. The patients were men and presented painless slow growing masses in the left cheek and hard palate, measuring $50 \times 30 \mathrm{~mm}$ and $23 \times 20 \mathrm{~mm}$ respectively. Microscopically, both lesions presented a solid proliferation of mature fat cells intermixed with bundles of connective tissue. Cells were immunopositive for S100 protein and CD34 (one case), with low mitotic activity (Ki-67). The final diagnosis was spindle cell lipomas. The lesions were excised and no recurrence was noticed after six months. Oral spindle cell lipomas are unexpected to occur in the oral mucosa, and the main differential diagnosis is well-differentiated liposarcoma/atypical lipoma. Lesions are treated with surgical excision and recurrences are rare.
\end{abstract}

Keywords: Lipoma; Mouth; Soft Tissue Neoplasms

\section{INTRODUCTION}

Lipomas are the most common soft tissue neoplasms; nevertheless the oral cavity is affected less frequently. According to the microscopic features, lipomas can be classified as conventional lipomas, fibrolipomas, angiolipomas, myxoid lipomas, sialolipomas, intramuscular lipomas, spindle cell/pleomorphic lipomas (SCPL), myolipomas, or chondroid lipomas [1-3]. Since the first report of an intra-oral SCPL, 23 new cases have been well documented $[1,2,4-9]$. So, we describe clinical and histological features of two new cases of intra-oral SCPL, and discuss the characteristics of this rare entity.

\section{CASE REPORT}

\subsection{Case 1}

A 38-year-old Caucasian man presented with a painless oral lesion that had been slowly growing for an indeterminate period of time. His medical history was noncomtributory, and an extra-oral examination showed no alterations. The tumor was located on the left cheek and was well circumscribed. It was pink-colored, with a smooth, lobulated, and nonulcerated surface. It was sessile with tender consistency and measured approximately 50 $\times 30 \mathrm{~mm}$ (Figure 1).

\subsection{Case 2}

A 60-year-old black man presented with a large asymptomatic mass on the center of the hard palate. According to the patient's report, the tumor had been slowly increasing in size for 38 years, though it did not interfere with his mastication or speech. No abnormalities were noticed from the extra-oral examination. An intra-oral examination revealed a unique, well-circumscribed, pedunculated, and pink-colored lesion, which was covered by healthy mucosa. The lesion had a firm consistency when palpated and measured $23 \times 20 \mathrm{~mm}$. The patient was a smoker and reported alcohol consumption. Additionally, he was a user of a partial removable prosthesis, though there was no history of chronic trauma.

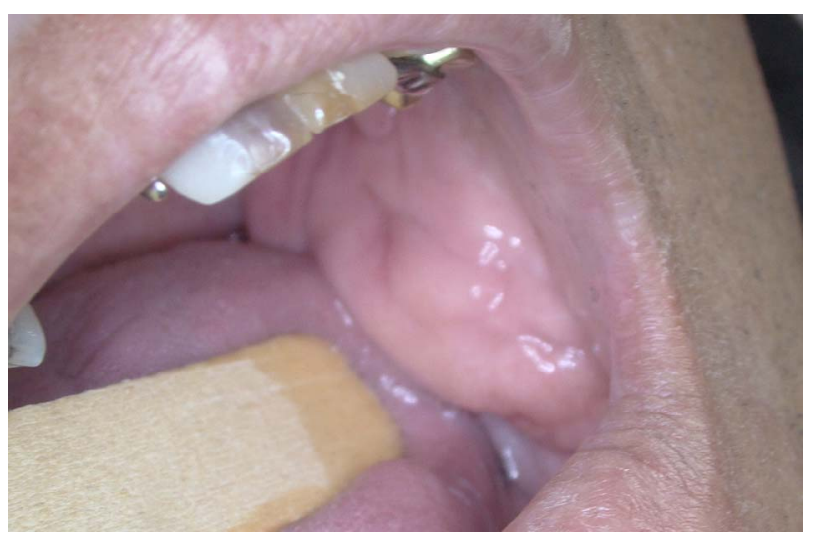

Figure 1. Clinical presentation of case 1: a well-circumscribed tumor in the left cheek, covered by nonulcerated mucosa. 


\subsection{Microscopic Findings}

In case 1 , an incisional biopsy was performed, while in case 2 the lesion was completely excised. Both procedures were performed under local anesthesia. Specimens were routinely processed and stained with hematoxylin and eosin (HE).

Microscopically, both lesions presented a solid proliferation of mature fat cells intermixed with bundles of connective tissue (Figure 2(a)). Fat cells showed a large univacuolated cytoplasm with a small, flattened nucleus in the periphery. Spindle cells with single, vesicular, oval or fusiform nuclei, as well as a poorly defined cytoplasm, were noticed to intermix with the fat cells. The underlying connective tissue presented short bundles of collagen (Figure 2(b)). Moreover, floret-like multinucleated cells could be observed (Figure 2(c)). Mitotic figures, nuclear pleomorphism, and hyperchromatism were absent. Vascularization and mast cells were evident, though lymphocytes were scarce. Spindle cells in case 1 were immunopositive for CD34 (Figure 2(d)), whereas the equivalent cells from case 2 were negative. Fat cells showed immunoreactivity for the S100 protein in both cases (Figure 2(e)). Additionally, Ki-67 immunostaining revealed low mitotic activity (not shown).

The final diagnosis for both cases was spindle cell/ pleomorphic lipoma. A complete excision was performed in case 1 . For both cases, no recurrence was noticed after six months of follow-up.

\section{DISCUSSION}

SCPL usually affects the posterior neck, shoulder, and back regions of middle-aged and elderly men [5]. Occurrence in the oral mucosa is uncommon. According to previous reported cases of oral SCPL and the present report, the most affected intra-oral sites are the tongue and buccal mucosa, although the floor of the mouth, lip, palate, and other regions may also be involved [1,2,4-9]. The male to female ratio is $1.7: 1$, and the mean age is 56.2 years. Oral SCPL usually presents as a solitary, wellcircumscribed, rubbery, painless, and slow-growing tumor, and it is covered by a healthy mucosa with sizes ranging from 3 to $50 \mathrm{~mm}$ (Table 1) [1,2,4-9]. The most affected intra-oral sites are the buccal mucosa and tongue, although the lip, floor of the mouth, palate, and other regions may also be involved. The male to female ratio is $1.36: 1$, and the mean age is 56 years [1,2,4-9]. Oral SCPL usually presents as a solitary, well-circumscribed, rubbery, painless, and slow-growing tumor, and it is covered by a healthy mucosa with sizes ranging from 3 to $50 \mathrm{~mm}[1,2,4-9]$.
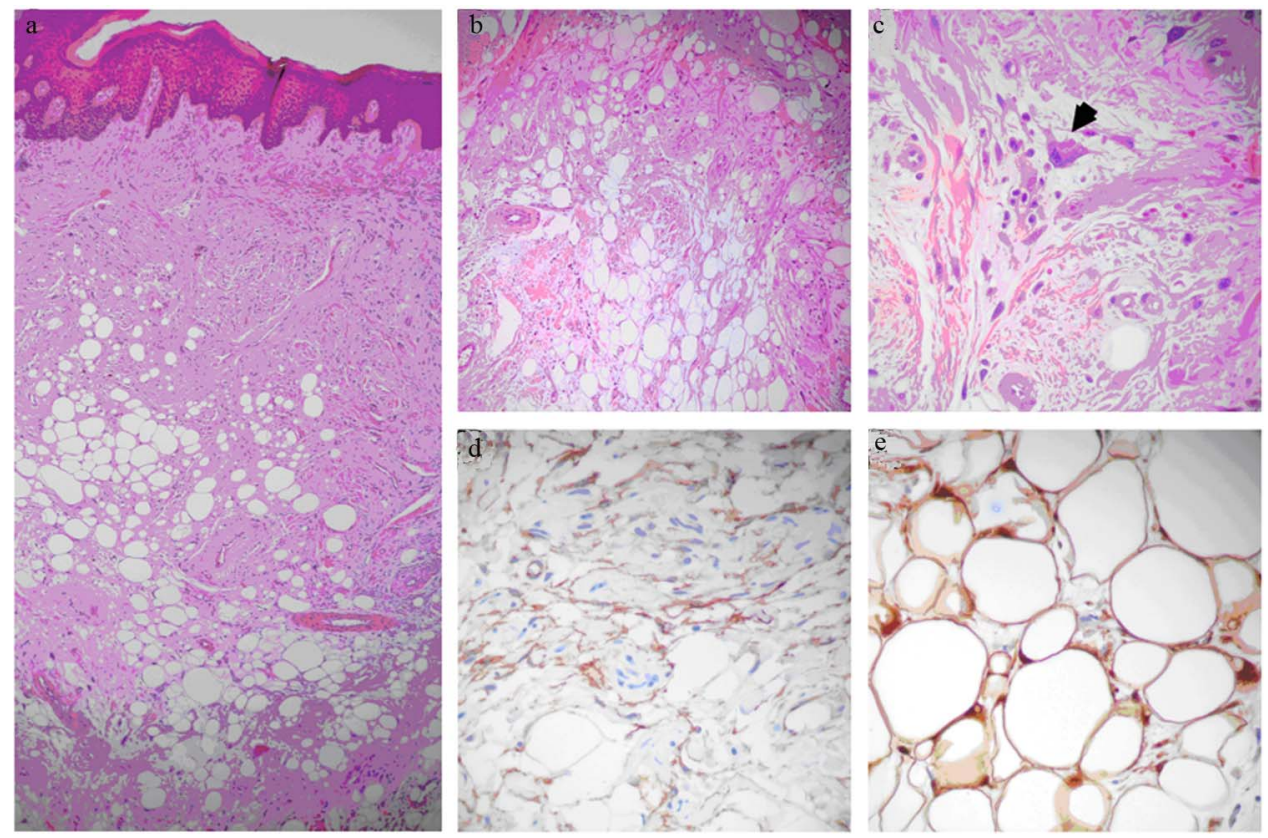

Figure 2. Microscopic features: (a) solid proliferation of mature fat cells intermixed with bundles of connective tissue (HE, $\times 50$ ); (b) Fat cells show a large univacuolated cytoplasm with a small flattened nucleus in the periphery. Spindle cells have single, vesicular, oval or fusiform nuclei, as well as a poorly defined cytoplasm. The underlying connective tissue presents short bundles of collagen (HE, ×100); (c) Floret-like multinucleated cell (arrow) $(\mathrm{HE}, \times 400)$; (d) spindle cells are immunopositive for CD34 (streptavidin-biotin, $\times 400$ ); (e) Fat cells show immunoreactivity for S100 protein (streptavidin-biotin, $\times 400$ ). 
Table 1. Features of the 27 cases of spindle cell lipoma reported in the oral cavity.

\begin{tabular}{|c|c|c|c|c|c|}
\hline Case number & Author/Year of publication & Site & Gender & Age (years) & Size $(\mathbf{m m})$ \\
\hline 1 & McDaniel et al., 1984 & Floor of mouth & Female & 33 & 10 \\
\hline 2 & McDaniel et al., 1984 & Tongue & Male & 52 & Not available \\
\hline 3 & Christopoulos et al., 1989 & Hard palate & Male & 58 & 20 \\
\hline 4 & Lombardi and Odell, 1994 & Tongue & Female & 63 & 15 \\
\hline 5 & Levy and Goding, 1989 & Floor of mouth & Female & 74 & 45 \\
\hline 6 & Tosios et al., 1995 & Cheek & Male & 55 & 40 \\
\hline 7 & Piattelli et al., 1999 & Cheek & Male & 75 & 20 \\
\hline 8 & Piattelli et al., 2000 & Cheek & Male & 63 & 25 \\
\hline 9 & Said-Al-Naief et al., 2001 & Tongue & Male & 66 & 30 \\
\hline 10 & Said-Al-Naief et al., 2001 & Tongue & Female & 53 & 7 \\
\hline 11 & Dutt et al., 1999 & Tongue & Female & 42 & 30 \\
\hline 12 & Khoo and Lian, 1995 & Cheek & Male & 23 & 50 \\
\hline 13 & Atik et al., 2002 & Tongue & Male & 45 & 20 \\
\hline 14 & Darling et al., 2002 & Alveolar mucosa & Male & 69 & 5 \\
\hline 15 & Piattelli et al., 2005 & Floor of mouth & Male & 50 & 10 \\
\hline 16 & Billings et al., 2006 & Lower lip & Female & 55 & 6 \\
\hline 17 & Billings et al., 2006 & Floor of mouth & Female & 84 & 10 \\
\hline 18 & Billings et al., 2006 & Cheek & Male & 88 & 10 \\
\hline 19 & Billings et al., 2006 & Tongue & Male & 45 & 9 \\
\hline 20 & Billings et al., 2006 & Tongue & Male & 67 & 10 \\
\hline 21 & Billings et al., 2006 & Tongue & Female & 31 & 3 \\
\hline 22 & Billings et al., 2006 & Tongue & Female & 75 & 5 \\
\hline 23 & Coimbra et al., 2006 & Floor of mouth & Female & 29 & 15 \\
\hline 24 & Imai et al., 2008 & Tongue & Male & 72 & 15 \\
\hline 25 & Vecchio et al., 2009 & Cheek & Male & 52 & 25 \\
\hline 26 & & Cheek & Male & 38 & 50 \\
\hline 27 & Caldeira et al., 2011 (present cases) & Hard palate & Male & 60 & 23 \\
\hline
\end{tabular}

Generally, clinical differential diagnoses include classic lipomas, fibromas, fibroepithelial polyps, benign nerve sheath tumors, salivary gland neoplasms, and herniated buccal fat pads [1]. Diagnostic hypotheses of case 1 comprised two categories: mesenchymal tumors of soft tissues (lipoma, neurilemmoma, neurofibroma) and nonneoplastic proliferative lesions (fibrous hyperplasia/traumatic fibroma). The most common location for oral lipomas is the buccal mucosa, and it was also considered because of the tender consistency of the lesion [4-6]. Considering the clinical aspects the herniated buccal fat pad, also called "traumatic pseudolipoma", must be included in differential diagnosis [1]. It presents as a sessile or pedunculated mass originated by trauma and is common in children but may also be present in adults. When it become epithelialized after trauma is not possible to distinguish from a true lipoma [1]. Because of clinical features, other lesions, such as oral dermoid, epidermoid cysts, and oral lymphoepitelial cysts should be considered in the differential diagnostic of oral lipomas [5]. In addition, in both cases the neurilemoma and neurofibroma were included in the differential diagnosis because of their similar clinical findings. In the case 1 , an incisional biopsy was performed due the extent of the lesion.

Referring to case 2, the differential diagnosis of the palatal mass includes the traumatic or irritation fibroma, benign and malignant salivary gland neoplasms, benign neural tumors and chronic abscess. These lesions have many characteristics in common and may appear clinically indistinguishable. Emphasis is placed on the importance of obtaining a thorough, comprehensive health and dental history and collecting relevant information. The period of duration of the lesion suggested a benign lesion. The hypothesis of chronic abscess was excluded after the dental clinical evaluation and the considered clinical diagnostic hypotheses were benign mesenchymal neoplasms, including neurilemmoma and neurofibroma, as considered in the former case, benign salivary gland tumor (pleomorphic adenoma), and fibrous hyperplasia/traumatic fibroma. Considering the clinical aspect and the long period of evolution of the lesion an excisional biopsy was performed in this case.

Imaging examination using ultrasonography, computed tomography, and magnetic resonance may aid the diagnosis of oral lipomas. Unfortunately, there is quite 
little information about specific imaging features of oral SCPL and we could not access images of the present cases. However, as mention above, SCPL is only a histopathological type of a lipoma, which may share imaging features with lipomas overall. On ultrasonography, oral lipomas are usually hypoechoic. On the computed tomography images, lesions have a density ranging from -123 to -83 Hounsfield units, with ill defined margins and with a characteristic homogeneous appearance with the same density as subcutaneous fat. Lesions tend to present high signal intensity on T1-weighted images with proportional reduction in signal intensity in T2. It has been stated that imaging by magnetic resonance can be useful in the delimitation of oral lipomas [10-12].

Microscopically, oral SCPL presents as a well-circumscribed but rarely encapsulated lesion composed of a mixture of mature adipocytes and spindle-shaped cells in a fibrocollagenous and/or myxoid stromal background. The proportion of lipomatous, spindle cell, and stromal components may vary from one case to another. Fat cells are univacuolated with a small peripheral nucleus. Atrophic changes in the adipocytes can be noticed, giving these cells a pseudo-lipoblastic appearance, though true lipoblasts are absent in SCPL. Spindle cells are usually uniform in size, shape and staining intensity, and they show pale-staining, vesicular, oval or compressed nuclei along with a sparse cytoplasm. The collagenous background is typically composed of thick, ropey bundles. Mitosis, cellular pleomorphism, and hyperchromatism are extremely rare or absent. Mast cells are usually found, and lymphocytes may be present as well. Moreover, multinucleated floret-like cells can be observed, and vascularity is often inconspicuous [1,2,5]. In the present cases, only few differences regarding the histopathological features could be observed. In case 1, the spindle cell component was more exuberant than the adipose component, while in case 2, both were equally present. Moreover, multinucleated floret-like cells, myxoid stromal areas, and some atrophic changes in the fat cells were observed only in case 2 .

The spindle cells usually show positivity for CD34 and vimentin, while the adipocytes express the S100 protein $[4,8]$. Nevertheless, no reactivity to CD34 could be observed in case 2. Mast cells can be detected by the immuno-expression of tryptase [8] and the Ki-67 index shows a low mitotic activity. Our immunohistochemical findings are in accordance with previously reported findings. Additionally, SCPL exhibits cytogenetic aberrations of chromosomes 13 and/or 16 [2,9]. Moreover, some authors have observed that SCPL expresses androgen receptors, pointing to a possible role of sex hormones on its pathogenesis [4]. Nevertheless, this could not be observed with oral SCPL [2].
The main histopathological differential diagnosis to be considered is well-differentiated liposarcoma/atypical lipoma, a rare intra-oral lesion, which may present as a slow growing and painless mass that is found mainly in the tongue and cheeks [2]. This distinction, though difficult, must be made in order to avoid overtreatment and a long-standing follow-up of the patient [2]. Some features, such as the well-circumscribed form; superficial location; slow growth; absence of lipoblasts; uniform spindle cells associated with mature, thick, and regular collagen bundles; sparse vascularity; and the absence of atypical cells are more likely to be present in SCPL than in well-differentiated liposarcomas/atypical lipomas $[1,8$, 9]. On the other hand, the most important characteristics needed to establish the diagnosis of a liposarcoma are the presence of variations in adipocyte size, atypical and enlarged adipocyte nuclei, and hyperchromatic stromal cells [13]. Moreover, spindle cells, if present, may show nuclear atypia and hypercromasia [13]. An immunohistochemical study is not contributory in this distinction [13].

Depending on the predominant microscopic component of the lesion, SCPL may resemble many other lesions. If the spindle cell component is prominent, fibrolipomas, neurilemmomas, neurofibromas, solitary fibrous tumors, nodular fasciitis, and malignant histiocytomas may be considered. Fibrolipomas are less cellular than SCPL and show large bundles of collagen. In cases where spindle cells present palisading nuclei, S100 staining may be useful in distinguishing SCPL from a neurilemmoma. The possibility of a lipomatous neurofibroma or even a neurofibroma can be ruled out by the absence of wavy nuclei in the spindle cells and their immunonegativity for the S100 protein in SCPL [5,6] Solitary fibrous tumors may also show spindle cells that are positive for CD34; however, fat cells are absent in this lesion $[8,9]$. In nodular fasciitis, despite the plump benign spindle cells, there usually is little collagen. Finally, malignant fibrous histiocytomas can present spindle cells and multinucleated giant cells, though pleomorphisms, typical and atypical mitoses, and storiform arrangements are observed as well [3].

On the other hand, SCPL, with localized spindle cells, may be similar to conventional lipomas and lipomatous hemangiopericytomas. In lipomatous hemangiopericytoma, the collagenized matrix and adipocytes are associated with prominent hemangiopericytoma-like vessels [3]. Finally, a highly myxoid matrix may pose some confusion with myxolipomas and myxoid liposarcomas. Both lesions lack the wiry collagen; additionally, myxolipoma lacks positivity for CD34, whereas myxoid liposarcoma shows evident lipoblastic differentiation [5].

Oral SCPL is treated with surgical excision and recur- 
rences are not expected [7].

\section{ACKNOWLEDGEMENTS}

This study was supported in part by grants from PRPq-UFMG; FAPEMIG, CAPES, and CNPq, Brazil. Dr. Silva and Dr. Bernardes are research fellows of CNPq.

\section{REFERENCES}

[1] Said-Al-Naief, N., Zahurullah, F.R. and Sciubba, J.J. (2001) Oral spindle cell lipoma. Annals of Diagnostic Pathology, 5, 207-215. doi:10.1053/adpa.2001.26973

[2] Vecchio, G., Amico, P., Caltabiano, R., Colella, G., Lanzafame, S. and Magro, G. (2009) Spindle cell/pleomorphic lipoma of the oral cavity. Journal of Craniofacial Surgery, 20, 1992-1994. doi:10.1097/SCS.0b013e3181bd2dad

[3] Barnes, L., Eveson, J., Reichart, P., Sidransky, D., Eds. (2002) Pathology and genetics of tumours of soft tissue and bone. World Health Organization Classification of Tumours, IARC Press, Lyon, 427.

[4] Atik, E., Usta, U. and Aydin, N.E. (2002) Pleomorphic lipoma of the tongue. Otolaryngol Head Neck Surg, 126, 430-431. doi:10.1067/mhn.2002.123919

[5] Billings, S.D., Henley, J.D., Summerlin, D.J., Vakili, S. and Tomich, C.E. (2006) Spindle cell lipoma of the oral cavity. American Journal of Dermatopathology, 28, 2831. doi:10.1097/01.dad.0000189615.13641.4b

[6] Coimbra, F., Lopes, J.M., Figueiral, H. and Scully, C. (2006) Spindle cell lipoma of the floor of the mouth. A case report. Medicina Oral Patologia Oral y Cirugia Bucal, 11, E401-E403.
[7] Darling, M., Thompson, I. and Schneider, J. (2002) Spindle cell lipoma of the alveolar mucosa: A case report. Oral Surgery, Oral Medicine, Oral Pathology, Oral Radiology \& Endodontics, 93, 171-173. doi:10.1067/moe.2002.120520

[8] Imai, T., Michizawa, M., Shimizu, H., Imai, T., Yamamoto, N. and Yura, Y. (2008) Bilateral multiple spindle cell lipomas of the tongue. Oral Medicine, Oral Pathology, Oral Radiology \& Endodontics, 106, 264-269.

[9] Piattelli, A., Perrotti, V., Fioroni, M. and Rubini, C. (2005) Spindle cell lipoma of the floor of the mouth: Report of a case. Auris Nasus Larynx, 32, 205-207. doi:10.1016/j.anl.2005.01.013

[10] Chikui, T., Yonetsu, K., Yoshiura, K., Miwa, K., Kanda, S., Ozeki, S. and Shinohara, M. (1997) Imaging findings of lipomas in the orofacial region with CT, US, and MRI Oral Medicine, Oral Pathology, Oral Radiology \& Endodontics, 84, 88-95.

[11] Bandéca, M.C., De Pádua, J.M., Nadalin, M.R., Ozório, J.E., Silva-Sousa, Y.T. and Da Cruz, P.D.E. (2007) Oral soft tissue lipomas: A case series. Journal of the Canadian Dental Association, 73, 431-434.

[12] Cappabianca, S., Colella, G., Pezzullo, M.G., Russo, A., Iaselli, F., Brunese, L. and Rotondo, A. (2008) Lipomatous lesions of the head and neck region: Imaging findings in comparison with histological type. Radiology Medicine, 113, 758-770. doi:10.1007/s11547-008-0258-5

[13] Nascimento, A.F., McMenamin, M.E. and Fletcher, C.D. (2002) Liposarcomas/atypical lipomatous tumors of the oral cavity: A clinicopathologic study of 23 cases. Annals of Diagnostic Pathology, 6, 83-93. doi:10.1053/adpa.2002.32375 